\title{
An Investigation into Client Attrition at a Professional Services Firm: The Case of a Content Marketing Agency in Cape Town
}

\author{
Pontsho Ramontsha \\ Management College of Southern Africa (MANCOSA) \\ 16 Samora Machel Street Durban, KwaZulu-Natal, South Africa
}

\begin{abstract}
Due to the limited research into client attrition within South Africa's content marketing industry, there is a scarcity of understanding with regards to the causal factors clients attribute to discontinuing services from an agency. The contribution of this paper is to evaluate this topic within a digital media company's operations for the strategic benefit oftheir customer relationship management (CRM). On a broader spectrum it will add to the limited information within business sciences research regarding agency-client relations. The general assumption regarding causes of client attrition in the business-to-business realm has largely been client dissatisfaction due to service failure. The research has sought to comprehend the current causes of client attrition and consequently formulate possible client retention strategies. The challenges in retaining its clients have been studied from a client's perspective. A number of practical recommendations were framed to halt the client attrition rate. These included introducing development and training initiatives for the agency's employees, as well as conducting more effective and frequent engagements between the clients and the agency's employees and senior management. It was found in the research that the agency's team should immerse itselfbetter in its clients' organisations to become more accustomed to the client's culture, operations and business targets.
\end{abstract}

Keywords: Client attrition, business targets, content marketing, media agencies

\section{INTRODUCTION}

A media agency based in Cape Town, South Africa, has experienced client attrition in recent years, resulting in a significant impact on the viability of the company. The research explores the challenges faced in retaining its clientsas losing them profoundly affects the organisation's performance. Content marketing is a modern media discipline that aims to attract and engage consumersthrough relevant, innovative content through various channels, which include published magazines, social media platforms, electronic newsletters, online blog posts and mobile device applications. Loss of clientele has resulted in the retrenchment of skilled, highly qualified employees and/or the restructure of the various divisions to adapt to the shrunken client base and impacting on the financial performance of the organisation. A disturbing level of uncertainty among employees is in evidence due to a perceived lack of employment security.Literature on managing client attrition in business-tobusiness relationships is relatively limited.This investigation is deemed important because a breaking of the agency-firm relationship is costly for both parties. The general assumption regarding causes of client attrition in the business-to-business realm has largely been client dissatisfaction due to service failure. In this regard, the role of the CAR is pivotal in formulating client retention strategies. It is important to understand the attributes of a favourable CAR from a client perspective, to identify the sources of dissatisfaction and how then the organisation can use this information to craft an impactful client retention strategy.The following questions are addressed in the study:

- What have been the reasons for clients cancelling their service contracts? What are current clients' perceptions of the service and product offerings?

- What strategies are recommended to improve customer retention?

At present there is no exhaustive study that analyses the causes of client attrition and little research into client churn within the broader industry of content marketing in South Africa. This study aims to contribute towards the production of such knowledge. 


\section{LITERARY REVIEW}

Diamond (2011:50) believes that the lion's share of profits will come from repeat clients, not from first-time clients. An effective business strategy involves ensuring a steady stream of repeat clients with new clients added into the fold. As content marketing is a service that is relatively modern in establishment, there is scant past literature on the topic. Much of the literature that is available deals with advertising agencies and focuses on client retention, with little attention paid to forerunning causes of attrition. Content marketing agencies - being the subject of this research - are classified as PSFs within the service industry. This sector has its own unique features that offer management challenges that are different from the traditional context. To understand the nature and operations of the agency, it is important to firstly understand these distinctive characteristics of professional services. From an academic research perspective, PSFs are a neglected yet vital economic sector (Trasorras, Weinstein and Abratt, 2009:616). From the available literature that reflects on what makes PSFs different, Loweldahl (2005:37-39) aptly summarised the characteristics in terms of five factors coined the five "I"s, being Interaction, Intangible, Individual judgement and local solutions, Innovation and Information asymmetry. Source: Loweldahl (2005:37-39)

Intangibility: Loweldahl (2005:37) refers to the fact that the inputs (which involve the methods, procedures and expertise of the individual professionals) and the outputs (the concepts and solutions) are intangible within PSFs. This is because the service involves a creative process. From this, it has been deduced that it could therefore be difficult to objectively evaluate quality in this sector. Interaction: Professional services are highly customised to each individual client and as such close cooperation between the two parties (the client and service provider) is required. To address that, the company has dedicated teams for each client that immerses themselves in the client's organisation as much as possible. This assists in coming up with a bespoke service offering that meets the unique needs of that particular client. Individual judgment and local solutions: Broderick (2010:12) puts forward that PSFs must be good at talent management. After all, people are the product. Broderick (2010:12) further states: "Without committed, highly skilled people, there is nothing to sell. And so people are treated with respect. Their opinions are valued, they are trusted to interact with clients early on in their careers, and their contributions are expected and rewarded." Client relationship management is an on-the-job training exercise for most employees. Innovation: Loweldahl (2005:39) explains that services delivered are highly idiosyncratic as the PSF largely comes up with specific solutions for each individual client. Therefore each client is so diverse it is impossible to create a onesize-fits-all solution.

Information asymmetry: Professional services are unique in that their value is as a result of superior knowledge in a certain field (Loweldahl, 2005:39); they are hired for their skills and experience. Broderick (2010:13) concurs and elaborates that successful firms invest significantly in training and mentoring their professionals. According to Broderick (2010:13) professionals are given a great deal of freedom, as long as they adhere to the values, and often are limited only by their ability and willingness to perform. Content marketing involves the creation and curation of text, photo and video content to keep customers and other stakeholders (employees, suppliers, the media, pundits, influencers, etcetera) continually engaged (Diaz, 2013:41). However, the marketing and communications industry (in all of its multifaceted formats) is transitioning through a period of marked, sometimes turbulent, change, forcing a re-examination of roles and responsibilities. There is also an explosion of other agencies suddenly claiming to offer contentmarketing. Advertisers and public relations agencies are all entering the field to contend for content marketing work with the specialist content marketers. This puts competitive pressure on specialist content marketers. Clients may opt to use the generalist agencies, such as an advertising agency purporting to provide content marketing services. Prince and Everett (2012:205-206), the reasons to end the arrangement can be categorised into a few dominant drivers: namely, the client-agency working relationship, service quality, and costs. One of the key client-agency literature studies available is West and Paliwoda (1996:22-39), which focuses on key attributes valued by clients.Today,service organisations have shifted their focus from transactional exchanges to relational exchanges to develop mutually satisfying relationship with customers (Coussement, 2014:477; Kotler and Keller, 2009:60). This is because extended relationships have a significant impact on profitability. A key goal for many organisations is to develop deep, enduring relationships with the people and organisations that could directly affect the firm's success (Kotler and Keller, 2009:60). Clients have an induction or orientation programme specifically designed for their consultants and contracted agencies to 
understand the nature of their world. Triki, A., Redjeb, N. and Kamoun, I. (2007:12) identified the client as a co-producer of the outcome and, indeed, this relationship of the client and agency is a relatively complex one, and in no way a traditional one (Loweldahl, 2005:44). When Triki et al. (2007:22) explored the causes of success or failure of the advertising agency-firm relationship; the research concluded that the relationship requires a high level of interactivity. Later research has also demonstrated that good CARs are important to client perceptions of the agency's work (Öhman, Häckner and Sörbom, 2012). The findings of Triki etal. (2007:15) identified role definition, ambiguity and incongruence as threats to the relationship. This close relationship is unavoidable. As more and more service providers are pushed from a service-centric focus into the practice of a client-centric strategy, many companies are turning to client relationship management (CRM) to better serve and facilitate closer relationships with clients (Coussement, 2014:477). Waugh and Grisson (2004:8) state that doing a good job is not enough to retain your clients, but rather a priority to strengthen relationships. Its recommendations to agencies include listening to your client, using the personal touch, asking them regularly how your agency are performing, learning about their problems and plans, and bringing your clients and your agency staff together often. The literature gives numerous explanations why agencies do not seem to be meeting their clients' expectations. The main reasons are that "Agencies oversell their abilities [because] agencies are insecure about their relationship with the client". Raising client expectations higher than what an agency can achieve is likely going to cause client disaffection.Researchers found that agencies were not concerned with determining or even acknowledging their clients' needs. For example, the research found none of the agency respondents "indicated awareness of client dissatisfaction with their work". This is partly because "agencies avoid systematic appraisal of the cause of dissatisfaction after they lose an account", hence, weaknesses in agency performance never get improved.Lack of communication is another very important reason why agency-client relationships fail. Each party constantly rated poor communication highly and failing to listen is the most frequent cause of account changes.The best explanation that the literature offers in terms of explaining why the CAR breaks down is unmet client expectations (Ghosh and Taylor, 1999:141). Value is another element that is in close relation to service quality and client expectations. According to Trasorras et al. (2009:621), value is subjective and dependent on who is making that determination. For example, some clients identify the least expensive product or service as a source of value, while others may say the same thing about the most expensive of those items. Creating perceived value for clients is nonetheless a key success factor for PSFs.Cost is an important factor to consider for this agency, especially with the turbulent economic environment currently gripping South Africa, along with much of the world's economies. Clients are largely cost-sensitive and are looking to get services with a limited budget. According to Prince and Everett (2012:193), the longevity of a CAR can create cost advantages and operational efficiencies for both the client and the agency. Financial considerations can be the initial motivator for entering into and investing in a CAR. For many businesses it is more cost effective to hire external expertise to provide certain contributions rather than to hire experts on a full-time basis. The business can then focus on its core function and allow the agency contracted to perform its specialist function. Additionally, costs can also tie a client to an agency. Client retention strategies: Customer retention involves ensuring repeat patronage, and as Jeng and Bailey (2012:1572) states, it is different from, though closely related to, brand loyalty and purchase behaviour. In exploring customer retention strategies for telecommunication firms, Jeng and Bailey (2012:1571) pose that while "customer satisfaction is an important part of the relationship between a provider and customer, the real value of customer satisfaction is shown in retention". Coussement (2014:478) states that a critical domain for improving companies' retention strategies involves client churn prediction, which can have serious implications for companies' cumulative profit. The literature on agency-client relationship suggests that it is a very complex topic with many differing opinions on what makes a successful or unsuccessful relationship and what are the drivers of attrition.

\section{Methodology}

Using the qualitative paradigm there is a concern with generating theories with rich, subjective data; this constitutes an interpretive-inductive approach (Saunders, M.N.K., Lewis, P. and Thornhill, A. 2012:31) Nieuwenhuis (2012:51) states that the methodology of qualitative research is concerned with understanding the processes and contexts, both social and cultural, which underlie behavioural patterns. The design of this study was exploratory in nature as the purpose was to investigate the 
relationship between variables. Exploratory research, according to Saunders et al. (2012:134), is not intended to provide conclusive findings but rather to have a better understanding of the problem. With that in mind, Saunders et al. (2012:134) advises that when conducting exploratory research, the researcher should be willing and able to change their direction as a result of revelations from new data and insights. Exploratory research typically examines new research questions on which little or no previous research has been done and leaves room for further researchers (Brown, 2006:43). Research strategy is based on the underlying philosophical assumptions of the researcher. From the variety of research strategies available from which to select, the researcher opted for the one most congruent with their philosophical assumptions and the one most suitable for generating the type of data required to address the research questions posed (Nieuwenhuis, 2012:70).

Case study: Nieuwenhuis (2012:75) states that case study research is a "systematic inquiry into an event or a set of related events which aim to describe and explain the phenomenon of interest". The case study is aimed at gaining greater understanding of the dynamics of a specific situation. The case study strategy comprises looking at a small group, project, institution or company to explore the factors that contributed to the characteristics of the case under investigation (Saunders et al. 2012:171). The case study strategy was used in this research. For this particular study, the phenomenon of client attrition is one that is complex with multiple variables of potential significance. The case study method was preferred as it is anchored in real-life situations, allows for multiple voices, and offers a magnifying effect to a global context through a single unit. Other organisations and researchers can learn vicariously through this single case while still having the prerogative to be selective about what applies or does not apply to their specific context. The population in this study comprised the decision-makers in the communications and marketing departments of all clients, past and present. The number of clients amounted to 16 companies located across South Africa. The sample for this study was then drawn from this population. For this research non-probability sampling was preferred. This subjective selection design was chosen to allow the researcher to deliberately capture a wide range of data from diverse groups of clients. Non-probability sampling better suited the research problem due to its in-depth analysis of the specific case rather than generalisation. Due to its manageability with regards to cost and time required to execute, this method was also deemed most practical. A purposive sampling technique was applied. The researcher, an employee of the company, has a working knowledge of the content marketing industry and service offerings. This aided the judgement of the researcher in the selection process. A pilot study was conducted using one respondent to assess whether the methods and instruments applied were appropriate and feasible. The pilot study data informed a refinement to the interview questions.

Data collection and research instrument: It is important to firstly state that in qualitative research the data collection and analysis are not two separate processes but rather a cyclical and iterative process (Nieuwenhuis, 2012:81). To select the appropriate data collecting and analysis techniques, a sound understanding of the methods and tools available is imperative. The interview method was selected to collect primary data that was then subjected to analysis. Interviews are prolific in data gathering, especially in descriptive research. This technique is unique as it collects data through direct verbal interaction and comes in various forms, from highly structured through to open-ended. The researcher chose this data collection instrument because when investigating a phenomenon of this complexity, direct interviews with managers or executives who know the details regarding the management and/or termination of the CAR will yield the most knowledge. The interviews were conducted telephonically or preferably face-to-face. Face-to-face interviews were preferable as they allowed the researcher to control the flow of primary data collection and the ability to conduct the interviews in a more in-depth manner. The researcher adopted an inductive, thematic analysis process to firstly categorise the data and thereafter identify themes and patterns that could then afford meaning to the text acquired. Braun and Clarke (2006:6) refer to thematic analysis as a qualitative analytical method for "Identifying, analysing and reporting patterns (themes) within data. The initial step of the thematic analysis involved reading the interview transcripts repeatedly and noting any areas of interest in relation to the research questions. Thereafter a process of coding was undertaken with the utilisation of the Nvivo software - each code identified a feature of the data that was of interest to the researcher. The final phase of the Thematic Analysis involved the final analysis of the themes and the drafting of the appropriate findings which provide sufficient evidence of the themes within the data and go beyond a description of the data to make a compelling argument in relation to the research questions. 
Validity and reliability: To ensure validity of the research, the use of interviews as the research instrument was tested with peers and the research supervisor to ensure its appropriateness. Reliability is therefore a concern every time a single observer is the source of data, because "we have no certain guard against the impact of that observer's subjectivity" (Babbie, 2010:158). To counter this, the predetermined questions of the interview were compiled to ensure that topics were tested and re-tested through differently posed questions with the same participant to identify correlations between the two queries.

Limitations of the study: This study was conducted subject to the following limitations:

- Access to participants was challenging, more specifically regarding those located in distant provinces. For the interviews, 4 of the 9 participants were located in Johannesburg, South Africa, whereas the interviewer was in Cape Town. This disallowed face-to-face interviews.

- With regards to the previous clients, determining the most suitable participants was challenging as there were no contact details available.

This study was conducted subject to the following delimitations:

- The participants were required to be in a management position with the authority to select service providers for the organisation.

- The participants had to be within the marketing and/or communications functions of the organisation to ensure they had working knowledge of the services provided.

Control of bias: To ensure as much objectivity as possible for the research, the researcher designed the research instrument to eliminate any use of sexist or racial stereotyping. The data gathering process also avoided any personal or private information being sought by the data collector. The participants were all made to respond to the same predetermined questions.

Ethical considerations: For this research all the relevant stakeholders were asked for permission to either access data or consent to participating in the research directly. Formal permission was obtained from the company. Consent to participate was transparently sought from prospective participants and an explanation of the context and objectives of the research was communicated clearly. The perception by some clients that the outcome of this study could be seen as a threat or negative influence to their relationship with the agency was identified as a risk that could hinder their objectivity in responding to questions. To address that concern, the confidentiality and anonymity of the participants was assured. To protect the anonymity of the participants in the research, the interview transcripts do not reveal the identity of participants and the letter sent to those taking part explained that information obtained during the course of the study was for research purposes only and would not in any way affect the respondents and their relationship with the company. Additionally, the responses of the participants were not attributed to an identifiable client during the data analysis process or in interpreting the findings. Participants were not misled or deceived during the research process and participation was voluntary.

\section{FINDINGS}

An inductive process was followed with themes being identified from the information obtained from clients of the agency that were purposely selected to garner a wide spectrum of experience with the agency. At various stages of the analysis, particularly at the stage of categorising the codes, modifications and adjustments of themes were necessary to present a rich, accurate and full picture of the data. A dominant theme that emerged from the data was the prioritisation of skills and service requirements. All but one of the nine interview respondents referred to, in some way, the importance of having an agency that can provide evidence of its capabilities and knowledge and ensure they fulfil their requirements as per the service contract. As stated by Loweldahl (2005:39), PSFs are unique in that their value is as a result of superior knowledge in a certain field and they are hired for their specialised skills and experience. Interpreting the respondent's stance on the matter, unmet requirements have an effect on the CAR and if the results are unfavourable this could lead to a cancellation of the business relationship. To differentiate the various factors that were revealed when discussing skills and requirements, three subcategories of this theme were detected: Innovation and market-leading knowledge; Capabilities and capacity; and Reputation. 
Innovation and market-leading expertise: Respondent 3, another current client who has been with the company for less than two years, also mentions how an innovative agency would assist their company to communicate and engage customers without diminishing their established brand identity. "I think we just need a bigger mix of ideas and experiences. We need to be able to really speak to our market in their language without diluting who we are. It's tricky. But... an agency like New Media has the professionals that know how to communicate to that market well."

Reputation: According to two of the respondents, Respondent 2 and Respondent 9 respectively, a track record of successful work executed is persuasive: "A track record is always quite important. I mean, if you can evidence your successes in a certain field..."

"Evidence is always the best pitch so we look for ...the, good track record."

A current long-term client, Respondent 4, clearly indicated: "But if the provider isn't living up to our expectations this would not stop us terminating the account." Respondent 5 declared: "We also want an agency that shows initiative. We have a lot on our plates a lot of the time so we can't be driving the process for them." This would call on the agency to anticipate the needs of the client rather than just being prescribed to.

The client-agency relationship: When analysing the research data collected, the significance of the $\mathrm{CAR}$ and its various dynamics has been a very prominent theme. Respondent 3 , in particular, articulated this important factor by stating: "We wouldn't be rash about terminating an agency's contract because we would have invested in that relationship, both in monetary terms and with our time and intellectual input."

Collaboration and interaction: Respondent 9 expressed this need for a productive relationship as follows: "We want an agency that we can forge a strong relationship with...if we can't work effectively together than would be an irreconcilable issue."

Communication and trust: In contrast to the subcategory aforementioned, 'collaboration and interaction', though the matter of communication and trust is not extensively dissected in the reviewed literature, it features prominently in the current research data.

"Well, at the initial phase it is always a little hard when both parties are getting to know each other. But honestly, as long as communication is good these are hurdles that can be overcome." This statement from Respondent 2, explains the crucial role that communication can play in the longevity of the business relationship.

Dedication and prioritising the client: The client in the relationship has a higher ranking role the agency must meet their needs. In this regard, it was noted from the data that at least one client had misgivings about how dedicated the agency was and more especially, how prioritised the client was by the senior management. Respondent 6 voiced these concerns:

"And I also would like more of the leadership to be involved as much as possible. I sometimes get the impression that the seniors that hook the account really are the ones most prominent and pitch phase and then fade into the background only to re-emerge at the next pitch. That's disconcerting. We need to know we are a priority, no matter the life stage of our contract." Though this is only one respondent that alluded to this nature of dissatisfaction, it puts into question whether the clients perceive the agency to have a client-centric approach.

Shared values and mission: According to Prince and Everett (2012:197), clients want their agencies to have sufficient knowledge regarding their particular organisation - including their circumstances, goals and values, as well as means of doing business. This understanding instils a level of confidence in the client that the agency appreciates their business objectives. Respondents expressed this outlook across the spectrum, be they current or previous clients. Speaking on this matter, current long-term client, Respondent 3 said: "I understand that our environments and cultures will be different. Ours is a financial services company so it will be different from a creative agency. But you can have different cultures and yet share the same values. The values are the important part. Like I said before our values are about innovation and excellence, those are things that we hold very seriously. And any agency that works with us has to have those elements. They have to illustrate those elements."

The client-agency misalignment: From the responses garnered, various respondents have described how a lack of fit between the agency and the current organisational requirements could result in the 
cancellation of the contract. Inversely, should the agency align with the needs of the organisation, this could translate to a lengthening of the contract. Respondent 6 , in explaining how they identify the "right" agency for their organisation, enlightened as follows: "We always have to make sure we are partnering with the best people for our cause... We look for values that can benefit our plan really well. So it would differ depending on what our business strategy is at the time. Sometimes we are looking for outrageous creativity that appeals to a certain group, other times we are looking for a firm that is specialising in a certain sector and does it well. It can be a case of using one agency to meet a certain need and another to answer another business requirement...So it's really out of the question to think we can do what they do. But there are a lot of other agencies that can do what they do. Our job is to choose the best one for us."

Selection: Literature has supported the notion that clients who dedicate significant focus to the selection process have been rewarded with successful relationships with their service providers (West and Paliwoda, 1996:22). This concept is one that is shared by a long-term client, Respondent 3, who specified: "The trick is always to pick the right agency at the get-go. Selection is very important. If you get that right, in my experience, the rest is not that hard."

Tailoring solutions: A prerequisite for PSFs, and most definitely very true of the company, the brand personalities of each client are so diverse it is impossible to create a one-size-fits-all solution (Loweldahl, 2005:39). Loweldahl's (2005:39) description of PSF services as "idiosyncratic" is concurred by Responded 3, who affirmed: "So maybe it is something that has worked for their other clients, but it shouldn't be a one-size fits all approach. That's another problem. Agencies sometimes have uniform solutions that they punt to each client. They may tweak it here and there, but it is the same thing. It doesn't work if the clients are quite different. That also tell me that an agency doesn't really know its clients if it does that."

Understanding each other's roles and needs: Described by some of the clients as a partnership, the alignment of the relationship between the agency and the client has been identified as an important factor in ensuring productive outputs for both parties. Encouragingly, some of the respondents of the study noted that this association required some understanding and appreciation from their side as well.

The role of costs: Cost constraints are well established in studies regarding client attrition; the data feedback itself obtains mention of company budgets and reducing cost. The clients are all commercial enterprises, which mean profits are a main priority and as such expenditure must be managed adequately. What is interesting though is that the respondents are not overtly unenthusiastic about spending money should the service be one of quality. Respondent 7 clearly stated that costs could be justified to their financial department if the service promises good returns that would validate the expenditure. "But we also make expert calls on things and if it will ultimately benefit or be to the detriment of the company, even the finance guys will put their calculators aside and listen to the experts in that field."

Clients' perceptions: The dominant view from respondents is a considerably positive one. This supports the theory previously presented that time is a positive influencer of the client agency relationship.

\section{Findings in relation to the reasons clients have for cancelling service contracts}

- Client attrition, or discontinuation of services, is strongly be linked to the agency not gaining sufficient knowledge of their client's brand, their goals and vision, and therefore, not meeting their requirements.

- From a client's perspective the most important aspect of the relationship between the agency and the client organisation is collaboration. The employees of the organisation have in-house knowledge and the agency has the required specialist skills, working together these two parties bring about favourable outcomes. If there is collaboration, as suggested by most respondents, rash decisions about terminating the contract are unlikely to occur.

- Client organisations look to their content marketing agency to be innovative and knowledgeable about current marketing trends. Clients have experienced disappointment when their agency misses strategic opportunities or does not leverage well off a trend to benefit the client's brand. In these cases is the client that has to then explicitly request or instruct the agency about a possible action 
plan. The client translates these incidents to mean that the content marketing agency does not fully understand the agency's needs and processes to effectively apply the trends or the agency is not cutting-edge in their service offering.

- Agencies have tended to over-commit and under-produce. Clients understand this to mean that the agency does not have adequate capacity to handle the work. The resulting concern by clients from this is that if the agency's employees are overworked, they will not be as dedicated as is required.

- When there is a perception by the client of a lack of dedication from the agency, especially with regards to understanding the processes, brand identity, image and organisational objectives, then conflict arises and the trust required to have faith in successful outcomes is damaged.

- Though a cooperative relationship is required, the relationship is not equal and the onus is on the agency to consistently show its worth to the client organisation at all stages of the relationship life cycle, not just at the agency selection phase.

- The clients value the monetary and intellectual investment they have made into the relationship with the agency and the service cost is only interrogated when the expectations about performance are not being met.

- If the switching costs are less costly than retaining the agency - then this will contribute to terminating the relationship. This is especially so if another agency can provide the same service at a lower service fee.

- Content marketing is a relatively new service offering and there is some resistance to change by members in the client organisations more accustomed to the traditional marketing tactics. These traditionalists do not understand that content marketing has a long-term approach with regards to benefits; they gravitate towards instant rewards and gratification and so do not have the patience for this new method that they do not really understand.

- What happens at the beginning of the CAR can significantly contribute to the ending of the relationship. The selection process is a vital process; a client that does is not careful to consider all the important objectives when selecting their agency can select an agency that in the long-term does not align well with its business objectives and termination of the relationship is then unavoidable.

- The longevity of the CAR can cause the agency to become complacent and produce substandard results, which clients are not willing to accept and will then seek out competing agencies for better performance and results.

\section{Findings in relation to current clients' perceptions as a content marketing agency:}

- In terms of skill-sets, clients' observation is that outsourcing services to the agency is beneficial as they have the specialist skills and by being an external party they are removed from the internal branding process itself and can give a qualified, outsider's perspective.

- Clients that are targeting new markets, more especially the younger consumer market and the emerging markets, are impressed with the agency's ability to communicate and engage these groups.

- There are clients who are disappointed with the way the agency has shown disregard towards the client organisation's values, mission, and brand legacy. This has created the impression that they are not a priority to the agency.

- Given that collaboration is vital for clients, the agency has not done well with regards to team integration. There is a perception by some clients that not all team members are equally involved as only a few representatives deal directly with the client. The lack on interaction produces an impersonal nature to the relationship that causes some distance between the agency and client.

- The fact that the agency has notable skills does not diminish the role of the client organisation's team of marketing and communication professionals. The opinion by some respondents is that the agency does not sufficiently seek guidance from internal employees who possess the "in-house" knowledge. The agency is seen as to some extent uncooperative and arrogant in its approach. 
Findings in relation to recommended strategies that could be employed to improve client retention at the agency

- The level of specialist skills is an important factor that clients assess an agency by and to instil confidence in a client, the company should consistently demonstrate these skills and focus on developing their staff's abilities and knowledge.

- Clients are impressed by innovation and agencies should exhibit this trait as much as possible. Important to note is this: even though innovation is required, it should not compromise the established brand identity and legacy. An agency should understand their client's heritage and work within that framework while still producing innovative solutions.

- Clients do not appreciate blanket approaches and generic solutions and services. Strategies and marketing campaigns need to be specifically tailored according to organisation's business environment, industry conditions and strategic objectives.

- Where there are differences of opinion it is imperative that the parties are able to talk about it and come to a mutual decision, and understanding. Communication is a key component to a longlasting business relationship.

- Agencies need to bear more awareness to costs when formulating marketing solutions as it is an important factor for all businesses. Without stifling the creativity, the service fees need to be sensible.

- Intellectual property is a cornerstone of communications and marketing and advertising, and an agency is viewed as a strategic partner in these fields. That is why an agency must be selective about possible clients and cannot be contracted by competing companies. This will raise many issues regarding conflict of interest.

- If an agency can demonstrate that it shares the values of its clients, it does not matter what the two entities' backgrounds are diverse. If it is established that the agency and client have shared goals and vision, the client will more readily trust that the agency can achieve optimal outcomes.

Research conclusions and recommendations: From all the data deciphered, specialist skills and collaboration are the overriding factors for clients of the company. The main conclusions from the study were in relation to expertise and relationship management and these challenges will form the basis for the majority of the recommendations. In general, employee development should be a key focus for the company to enhance its capability and consequently retain its clients. The agency must ensure that its employees are continually trained in all technical aspects and also kept abreast of the latest industry trends and innovations. The emphasis should be placed on continuous learning as the discipline and field of content marketing is a rapidly evolving one, more especially with regards to technological advances. Training should also be provided specifically on client relationship management to all client-facing employees. To accomplish this there needs to be a clear instruction to the agency's human resource (HR) function to compile an HR strategy that will include assessing employees' current abilities and thereafter provision of the required training. More dedication must be placed on thoroughly understanding clients' environments, including their cultures, processes, operations and objectives. The agency's senior management should also engage more with the clients. This will also highlight to the clients that they are prioritised and have a dedicated team exclusively working for them. A number of attributes were found to be pivotal themes in this explorative study, all of which were judged to be conduits for a successful CAR. Of these the most stressed were those relating to competencies and methods of working. The ideal agency for the majority of respondents would be an innovative, collaborative firm that was well versed on who its client is and what their mission and vision is. From these discoveries a number of practical recommendations were offered for the agency to possibly improve its competitive edge. The main recommendations were in relation to development and training as well as more effective engagements. The HR operation of the agency would have to take the lead with much of the training and development suggestion, while having the full support of senior management. The nature of engagement is one that will also require senior management will and introspection. The agency needs to make changes to ensure that both perception and reality evidences the agency as having a strong client-centric culture. All these findings from the study should prove valuable for further study. This research has, by its undertaking, exposed the lack 
of exploration with regards to the challenges and nature of the creative agency and its relation with its clients. This void in business research warrants more exploration and this study is a stimulating basis for future research.

\section{REFERENCES}

[1] Babbie, E. R. (2010) The Practice of Social Research. Cengage Learning. p. 150-167.

[2] Braun, V. and Clarke, V. (2006). Using thematic analysis in psychology. Qualitative Research in Psychology. [online] p. 77-90. Available from: http://www.informaworld.com/smpp/content $\mathrm{db}=\mathrm{all} \sim$ content $=\mathrm{a} 795127197 \sim$ frm $=$ titlelink

[3] Broderick, M. (2010). Professional services: Characteristics, challenges and leadership model. Art of Managing Professional Services: Insights from Leaders of the World's Top Firms. FT Press. Chapter 1.

[4] Coussement, K. (2014). Improving customer retention management through cost-sensitive learning. European Journal of Marketing. 48(3/4), p. 477-480. Emerald Group Publishing Limited.

[5] Diamond, M. (2011). Are you a client killer? Phones not ringing? Maybe you drove them away. Reeves Journal: plumbing, heating, cooling. June 2011, p. 50-51.

[6] Diaz, A. (2013). Welcome to content marketing. Caribbean Business, Puerto Rico. 15 August 2013, p. 41.

[7] Ghosh, B.C. and Taylor, D. (1999). Switching advertising agency: a cross-country analysis. Marketing Intelligence and Planning. 17(3), p. 140-148. MCB University Press.

[8] Jeng, D.J. and Bailey, T. (2012). Assessing customer retention strategies in mobile telecommunications. Management Decision. 50(9), p. 1570-1595. Emerald Group Publishing Limited.

[9] Kotler, P and Keller, K.L. (2009). Marketing Management (13th Edition). Upper Saddle River, New Jersey: Prentice Hall.

[10] Loweldahl, B.R. (2005) Strategic Management of Professional Firms. (Third Edition). Copenhagen Business School Press.

[11] Nieuwenhuis, J. (2012) Qualitative Research. In Maree, K. (2012). First Steps in Research. (11th Edition). Van Schaik Publishers. Pretoria, South Africa.

[12] Öhman, P., Häckner, E. and Sörbom D. (2012). Client satisfaction and usefulness to external stakeholders from an audit client perspective. Managerial Auditing Journal. 27(5), p. 477-499.

[13] Prince, M. and Everett, R.F. (2012). Switching costs: A key to understanding and managing business consulting relationship longevity. Business-to-Business Marketing Management: Strategies, Cases and Solutions. 18, p. 193-217. Emerald Group Publishing Limited.

[14] Saunders, M.N.K., Lewis, P. and Thornhill, A. (2012). Research Methods for Business Students (Sixth edition). Harlow, England: Financial Times, Prentice Hall.

[15] Trasorras, R., Weinstein, A. and Abratt, R. (2009). Value, satisfaction, loyalty and retention in professional services. Marketing Intelligence and Planning. 27(5), p. 615-632. Emerald Group Publishing Limited.

[16] Triki, A., Redjeb, N. and Kamoun, I. (2007). Exploring the determinants of success/failure of the advertising agency-firm relationship. Qualitative Market Research: An International Journal. 10(1), p. 10-27. Emerald Group Publishing Limited.

[17] Waugh, T.A. and Grissom, A.T. (2004). Keeping your clients sold. Accounting Today. 5-18 April 2004.

[18] West, D.C. and Paliwoda, S.J. (1996). Advertising client-agency relationships: The decisionmaking structure of clients. European Journal of Marketing.30 (8), p. 22-39. MCB University Press. 